\title{
A composite bosons minimal basis for the pairing Hamiltonian
}

\author{
M.B.Barbaro ${ }^{1}$, A.Molinari ${ }^{1}$, F.Palumbo ${ }^{2}$ and M.R.Quaglia ${ }^{1}$ \\ ${ }^{1}$ Dipartimento di Fisica Teorica dell'Università di Torino and INFN \\ Sezione di Torino, via P.Giuria 1, I-10125, Torino, Italy \\ ${ }^{2}$ INFN - Laboratori Nazionali di Frascati,P.O.Box 13, I-00044 Frascati, \\ Italy
}

\begin{abstract}
The pairing interaction among identical nucleons in a singleparticle level is treated in the hamiltonian formalism using even Grassmann variables. A minimal (irreducible) basis having a remarkable symmetry property is set up using composite, commuting variables with a finite index of nilpotency. Eigenvalues and eigenfunctions of given energy, seniority and zero third component of the angular momentum of the pairing hamiltonian are then found. The eigenvectors, which cannot be cast solely in terms of composite bosons with angular momentum zero and two, are expanded in the minimal basis with coefficients analytically expressed in terms of a generalized hypergeometric function.
\end{abstract}

PACS: 24.10.Cn, 21.60.-n

Keywords: Grassmann algebra; Nuclear pairing interaction; Bosonization.

Corresponding author: Maria B. Barbaro, Dipartimento di Fisica Teorica, Università di Torino, Via P. Giuria 1, I-10125 Torino - Italy, Tel: 39-116707212, Fax: 39-11-6707214, e-mail: barbaro@to.infn.it 
A difficulty inherent to nuclear physics relates to the number of nucleons in a nucleus which is not large enough to exploit the field theoretical techniques as successfully as in condensed matter physics, but, on the other hand, is large enough to require a high dimensionality for the basis in which the physics of the nucleus is treated.

The standard approach to overcome this obstacle reduces the size of the basis by selecting the relevant degrees of freedom, namely those appropriate for the description of the low-lying nuclear excitations, and then by introducing the associated variables, for example as done in the Arima and Iachello model [1].

Clearly one would like to devise a systematic procedure to carry out this reduction starting from an underlying, more fundamental hamiltonian expressed in terms of fermionic degrees of freedom.

Recently we have indeed tried an approach [2] to this problem in the path integral framework by performing a non-linear change of variables in the Berezin integral defining the partition function of a fermionic system, the new integration variables then representing composite bosons of appropriate quantum numbers.

As a simple example, we have applied this method to the problem of an even number $N$ of nucleons sitting in a single-particle level with angular momentum $j$ (third component $m$ ) and interacting through the well-known pairing hamiltonian $H_{P}$. We have been able to express the pairing action and the ground state wave function with zero seniority in terms of the composites and to recover the familiar formula for the ground state energy of $H_{P}$ [2]. Moreover through this study we learned about several useful properties of these composites which have been applied to set up a new perturbative expansion in QCD [3]- 国].

However, to solve the problem of the excited states of $H_{P}$ (those with nonzero seniority $v$ ) and to extend the approach to encompass the long range quadrupole-quadrupole force as well as the pairing interaction has proven to be a hard task to perform. Therefore in this letter we address the $v \neq 0$ problem without resorting to the change of variables in the Berezin integral. Instead we explore whether and how the Fock basis of $H_{P}$, set up with determinants of single-particle states, can be reduced to a minimal dimension and whether the minimal basis can be expressed in terms of composite bosons. Indeed one might expect the physics of $H_{P}$, which only acts among pairs of nucleons coupled to angular momentum $J=J_{z}=0$, to be describable by 
only two composite bosons having the two constituent nucleons coupled to $J=0$ (the first) and to any allowed non-vanishing angular momentum (the second).

Specifically, in carrying out the program of reducing the natural Fock basis of $H_{P}$ we shall search for a minimal (irreducible) basis, fully solving the pairing problem, using building blocks associated with composite, commuting variables having a finite index of nilpotency. Hence these are not to be viewed as bona fide bosons, being made up of fermions satisfying the Pauli principle; and this is why we shall refer to them as composite bosons. Notably the dimensions of the basis will turn out to be fixed by the number $n=N / 2$ of pairs present in the problem.

To illustrate how our approach works, let us start by recalling that in the quasi-spin scheme one introduces operators annihilating (creating) pairs of particles in orbits having appropriate time-reversal properties, i.e. of the type

$$
\hat{B}_{J}=\sqrt{\frac{\Omega}{2}} \sum_{m=-j}^{j}\langle j m, j-m \mid J 0\rangle \hat{a}_{-m} \hat{a}_{m}
$$

(and the hermitian conjugate). In the above $\langle j m, j-m \mid J 0\rangle$ is the usual Clebsch Gordan coefficient and $\Omega=(2 j+1) / 2$. In terms of (1) the pairing hamiltonian reads

$$
\hat{H}_{P}=-g_{P} \hat{B}_{0}^{\dagger} \hat{B}_{0} .
$$

As mentioned above, one might conjecture that the eigenstates of $H_{P}$ could be set up in terms of the operators $\hat{B}_{0}$ and $\hat{B}_{J}$ where the specific value of the index $J(\neq 0)$ in the latter should be irrelevant, since any $J \neq 0$ corresponds to a broken pair. For example, in the spirit of the Arima and Iachello model, one could set $J=2$.

However in the framework of the creation and annihilation operators the commutator

$$
\left[\hat{a}_{-m} \hat{a}_{m}, \hat{a}_{m^{\prime}}^{\dagger} \hat{a}_{-m^{\prime}}^{\dagger}\right]=\delta_{m m^{\prime}}\left(1-\hat{a}_{m^{\prime}}^{\dagger} \hat{a}_{m}-\hat{a}_{-m}^{\dagger} \hat{a}_{-m^{\prime}}\right),
$$

which includes, e.g. for $J=J^{\prime}=0$,

$$
\left[\hat{B}_{0}, \hat{B}_{0}^{\dagger}\right]=\Omega\left(1-\frac{\hat{n}}{\Omega}\right),
$$


is non-canonical, thus rendering non-trivial the task of finding the eigenstates of $H_{P}$ ( $\hat{n}$ is the fermion number operator).

An approach that circumvents this difficulty is the hamiltonian framework employing Grassmann variables [5. Here one exploits the isomorphism between the Fock space $F$ generated by a set of $N$ fermionic creation operators $\hat{a}_{1}^{\dagger}, \cdots \hat{a}_{N}^{\dagger}$ and the so-called $\mathcal{G}^{+}$algebra built with the set of anticommuting objects $\lambda_{1}^{*}, \ldots \lambda_{N}^{*}$ (the generators of the algebra). The isomorphism is defined by mapping the vectors $\hat{a}_{1}^{\dagger} \ldots \hat{a}_{j}^{\dagger} \mid 0>$ onto the elements $\lambda_{1}^{*} \ldots \lambda_{j}^{*}$. The image of a generic vector $\mid \Psi>\in F$ under this mapping will be denoted by $\Psi\left(\lambda^{*}\right)$. Next, to a linear operator in normal form,

$$
\hat{\mathcal{O}}=\sum_{i_{1} \ldots i_{k}} \sum_{j_{1} \ldots j_{k}} \mathcal{O}^{i_{1} \ldots i_{k}, j_{1} \ldots j_{k}} \hat{a}_{i_{1}}^{\dagger} \ldots \hat{a}_{i_{k}}^{\dagger} \hat{a}_{j_{1}} \ldots \hat{a}_{j_{k}},
$$

is associated the following function of the Grassmann variables

$$
\mathcal{O}\left(\lambda^{*}, \lambda\right)=\sum_{i_{1}, \ldots i_{k}, j_{1} \ldots j_{k}} \mathcal{O}^{i_{1}, \ldots i_{k}, j_{1} \ldots j_{k}} \lambda_{i_{1}}^{*} \ldots \lambda_{i_{k}}^{*} \lambda_{j_{1}} \ldots \lambda_{j_{k}},
$$

which allows one to define the kernel

$$
K_{\mathcal{O}}\left(\lambda^{*}, \lambda\right)=\mathcal{O}\left(\lambda^{*}, \lambda\right) \mu_{+}\left(\lambda^{*} \lambda\right) .
$$

Then the action of $\hat{\mathcal{O}}$ on a state $\Psi$ reads

$$
(\hat{\mathcal{O}} \Psi)\left(\lambda^{*}\right)=\int\left[d \lambda^{\prime *} d \lambda^{\prime}\right] K_{\mathcal{O}}\left(\lambda^{*}, \lambda^{\prime}\right) \mu_{-}\left(\lambda^{\prime *} \lambda^{\prime}\right) \Psi\left(\lambda^{\prime *}\right) .
$$

In the above

$$
\mu_{ \pm}\left(\lambda^{*} \lambda\right)=e^{ \pm \sum_{i} \lambda_{i}^{*} \lambda_{i}}
$$

In this framework the pairing hamiltonian is then readily expressed in terms of the even, nilpotent, commuting Grassmann variables

$$
\varphi_{m}=(-1)^{j-m} \lambda_{-m} \lambda_{m}
$$

according to

$$
H_{P}=-g_{P} B_{0}^{*} B_{0} \quad \text { with } \quad B_{0}=\sum_{m=1 / 2}^{j} \varphi_{m} .
$$

Now, in seeking a reduction of the basis dimensionality, we shall be guided by the two main features of $H_{P}$, namely that it 
1. is expressed solely in terms of the $\varphi$,

2. is invariant for any permutation of the $\varphi$.

These properties suggest also expressing the vectors of the basis in terms of the variables (10). For this scope, the action of $H_{P}$ on the latter should be explored.

This can be accomplished along the lines illustrated in [2], the result being

$$
H_{P} \psi\left(\varphi^{*}\right)=\int\left[d \eta^{*} d \eta\right] K_{P}\left(\varphi^{*}, \eta\right) e^{\sum \eta^{*} \eta} \psi\left(\eta^{*}\right)=E \psi\left(\varphi^{*}\right)
$$

where the integral is over the even elements of the Grassmann algebra as defined in [2] and the kernel is

$$
K_{P}\left(\varphi^{*}, \eta\right)=H_{P}\left(\varphi^{*}, \eta\right) e^{\sum \varphi^{*} \eta} .
$$

Hence, the above-mentioned difficulty associated with the non-canonical nature of the commutator (3) disappears.

We then attempt to diagonalize the $H_{P}$ associated with $n=N / 2$ pairs in a basis spanned by states represented as products of $n$ factors $\varphi^{*}$ 's, namely of the type

$$
\varphi_{m_{1}}^{*} \cdots \varphi_{m_{n}}^{*} .
$$

Since the number of states here is clearly $\left(\begin{array}{l}\Omega \\ n\end{array}\right)$, the very large reduction of the basis dimension entailed by the choice of the variables (10) is apparent: indeed in terms of fermionic degrees of freedom, the corresponding basis would have a dimension $\left(\begin{array}{l}2 \Omega \\ 2 n\end{array}\right)$.

Moreover, because the variables $\lambda$ anticommute, each vector of the basis (14) is antisymmetric with respect to the exchange of any pair of fermions, and hence the above basis fulfills the Pauli principle.

We now explore whether, for a given $\Omega$ and $n$, the eigenstates of $H_{P}$ can be cast in the form

$$
\psi\left(\varphi^{*}\right)=\sum_{m=1}^{\left(\begin{array}{l}
\Omega \\
n
\end{array}\right)} \beta_{m}\left[\varphi_{m_{1}}^{*} \cdots \varphi_{m_{n}}^{*}\right]_{m}
$$

the index $m$ identifying the set $\left\{m_{1}, m_{2} \cdots m_{n}\right\}$ and the $\beta_{m}$ being complex coefficients. 
In the simple situation where $n=1$, the states in (15) are indeed eigenstates of the pairing hamiltonian. In fact the eigenvalue equation, with $\mathcal{E}=E / g_{P}$, is

$$
(\mathcal{E}+1) \beta_{m}+\sum_{p(\neq m)=1 / 2}^{j} \beta_{p}=0
$$

and in the basis in (14) the operator $\mathcal{H}_{P}=-\frac{H_{P}}{g_{P}}+\mathcal{E}$ is represented by a matrix of dimension $\Omega$ filled by ones but for the principal diagonal, whose elements are given by $\mathcal{E}+1$. This matrix is invariant under any permutation of the index $m$ which labels its rows and columns. Moreover, of the $\Omega$ real roots of the associated characteristic equation

$$
(\mathcal{E}+\Omega) \mathcal{E}^{\Omega-1}=0
$$

only two are distinct, namely the lower $\mathcal{E}_{0}(n=1)=-\Omega$ with multiplicity $\delta=1$ and the upper one $\mathcal{E}_{2}(n=1)=0$ with multiplicity $\delta=\Omega-1$. The associated orthogonal eigenvectors are

$$
\Psi_{0}(n=1)=\frac{1}{\sqrt{\Omega}} \sum_{m=1 / 2}^{j} \varphi_{m}^{*}
$$

and

$$
\Psi_{2}(n=1)=\mathcal{N} \sum_{m=1 / 2}^{j-1} \beta_{m}\left[\varphi_{m}^{*}-\varphi_{j}^{*}\right]
$$

$(\mathcal{N}$ is a normalization factor). Notice that into the eigenvector in (18) enter $\Omega-1$ parameters (the $\beta_{m}$ ), which correspond to the degeneracy of the eigenvalue. The above eigenvalues agree with the quasi-spin formalism and correspond to the states with seniority $v=0$ and $v=2$ respectively, $v$ being an even non-negative number representing the number of unpaired particles.

Since the case with $n=\Omega-1$ is identical, but for a shift in energy, to the one with $n=1$ pairs and, by extension, the case with $n$ pairs is equivalent to the one with $\Omega-n$ pairs, in the following we shall confine ourselves to consider only $n \leq \frac{\Omega}{2}$.

When $n=2$, it is not so trivial to prove that (15) is an eigenfunction of the pairing hamiltonian for any $\Omega$, although this turns out to be the case. For example when $\Omega=4$ the eigenvalue equation is

$$
(\mathcal{E}+2) \beta_{m n}+\sum_{p(\neq n, m)=1 / 2}^{j}\left(\beta_{p m}+\beta_{p n}\right)=0
$$


and $\mathcal{H}_{P}$ is represented by the $6 \times 6$ matrix whose elements are $\mathcal{E}+2$ on the principal diagonal, 0 on the secondary diagonal and 1 elsewhere. The associated characteristic equation

$$
(\mathcal{E}+6)(\mathcal{E}+2)^{3} \mathcal{E}^{2}=0
$$

has three real distinct roots out of six, namely the lowest $\mathcal{E}_{0}(n=2)=-6$ with degeneracy $\delta=1$, the intermediate $\mathcal{E}_{2}(n=2)=-2$ with degeneracy $\delta=3$ and the highest one $\mathcal{E}_{4}(n=2)=0$ with degeneracy $\delta=2$.

The corresponding orthogonal eigenvectors are

$$
\begin{aligned}
\Psi_{0}(n= & 2)=\mathcal{N}_{0}\left(\varphi_{1 / 2}^{*} \varphi_{3 / 2}^{*}+\varphi_{1 / 2}^{*} \varphi_{5 / 2}^{*}+\varphi_{1 / 2}^{*} \varphi_{7 / 2}^{*}\right. \\
& \left.+\varphi_{3 / 2}^{*} \varphi_{5 / 2}^{*}+\varphi_{3 / 2}^{*} \varphi_{7 / 2}^{*}+\varphi_{5 / 2}^{*} \varphi_{7 / 2}^{*}\right) \\
\Psi_{2}(n= & 2)=\mathcal{N}_{2}\left[a_{1}\left(\varphi_{1 / 2}^{*} \varphi_{3 / 2}^{*}-\varphi_{5 / 2}^{*} \varphi_{7 / 2}^{*}\right)+a_{2}\left(\varphi_{1 / 2}^{*} \varphi_{5 / 2}^{*}-\varphi_{3 / 2}^{*} \varphi_{7 / 2}^{*}\right)\right. \\
& \left.+a_{3}\left(\varphi_{1 / 2}^{*} \varphi_{7 / 2}^{*}-\varphi_{3 / 2}^{*} \varphi_{5 / 2}^{*}\right)\right] \\
\Psi_{4}(n= & 2)=\mathcal{N}_{4}\left[b_{2}\left(\varphi_{1 / 2}^{*}-\varphi_{7 / 2}^{*}\right)\left(\varphi_{5 / 2}^{*}-\varphi_{3 / 2}^{*}\right)\right. \\
& \left.+b_{3}\left(\varphi_{1 / 2}^{*}-\varphi_{5 / 2}^{*}\right)\left(\varphi_{3 / 2}^{*}-\varphi_{7 / 2}^{*}\right)\right]
\end{aligned}
$$

$\mathcal{N}_{0}, \mathcal{N}_{2}, \mathcal{N}_{4}$ being normalization factors and $a_{1}, a_{2}, a_{3}, b_{2}$ and $b_{3}$ free parameters.

The case just discussed asks for some comments. First, as for $n=1$, the matrix representing $\mathcal{H}_{P}$ can be written in $\left(\begin{array}{l}4 \\ 2\end{array}\right)$ ! equivalent ways (not all distinct), each one corresponding to a different labelling of the states, but leading to the same determinant.

Furthermore in the above example the correspondence between the number of parameters that enter into a given wave function and the degeneracy of the corresponding eigenvalue is again apparent. In general to an eigenvalue $\mathcal{E}_{v}(n)$ of given seniority $v$ correspond [6]

$$
\delta_{v}=\left(\begin{array}{c}
\Omega \\
\frac{v}{2}
\end{array}\right)-\left(\begin{array}{c}
\Omega \\
\frac{v}{2}-1
\end{array}\right)
$$

independent states constructed using the building blocks (10) (in our convention a binomial coefficient with a negative lower index vanishes). The $\delta_{v}$ is commonly referred to as the seniority degeneracy. 
Finally to address the general case we exploit the two lessons learned from the previous example. The first one concerns the seniority degeneracy: its very existence proves that a further reduction of the dimension of the basis can be achieved. The second is that the structure of the symmetric matrix of dimension $\left(\begin{array}{l}\Omega \\ n\end{array}\right)$ associated with $\mathcal{H}_{P}$ should be *

$$
\left(\begin{array}{ccc}
\mathcal{E}+n & & 0 \vee 1 \\
& \ddots & \\
0 \vee 1 & & \mathcal{E}+n
\end{array}\right),
$$

where the symbol $0 \vee 1$ indicates that the upper (lower) triangle of the matrix is filled with zeros and ones. Indeed the matrix elements of $\mathcal{H}_{P}$ are one when the bra and ket differ by the quantum state of one (out of $n$ ) pair, otherwise they vanish. The diagonal matrix elements simply count the number of pairs.

An elementary combinatorial analysis then shows that the number of ones in each row (column) of the matrix (24) is given by $n(\Omega-n)$. Indeed a nonvanishing matrix element has the row specified by $n$ indices whereas, of the indices identifying the column, $n-1$ should be extracted from those fixing the row in all possible ways, which amounts to $n$ possibilities. The missing index should then be selected from among the remaining $\Omega-n$ ones: hence the formula $n(\Omega-n)$ follows.

To write down explicitly the matrix (24) it is convenient to divide the set of the $\Omega$ even Grassmann variables, whose quantum numbers identify the levels where the $n$ pairs are to be placed, into two subsets, one with $\Omega-\nu$ and the other with $\nu$ elements (to be referred to as I and II, respectively) with $n \leq \nu \leq \Omega-n$.

A priori each of these partitions is valid in the sense that it leads to a basis with a dimension lower than the one of (14). On the other hand the physics of the pairing hamiltonian is such that for a system of $n$ pairs $n+1$ eigenvalues should be expected, no matter what the value of $\Omega$. They of course correspond to the breaking of $0,1,2 \cdots n$ pairs. A partition leading to an $n+1$ dimensional basis, for any $\Omega$ and without degeneracy, is the one which has $\nu=n$.

Indeed in this instance the $\left(\begin{array}{l}\Omega \\ n\end{array}\right)$ entries of each row and column of the matrix can then be grouped into $n+1$ sets, the first one corresponding to

\footnotetext{
*As already noticed in the $n=2$ case, all of the $\left(\begin{array}{l}\Omega \\ n\end{array}\right)$ ! orderings of the states are equivalent.
} 
the $n$ pairs placed in the $\Omega-n$ levels of I, the remaining $n$ levels of II being empty (see Fig. 1a).

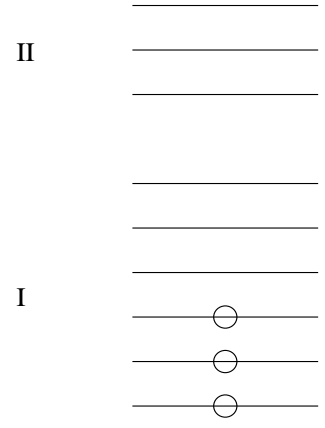

(a)

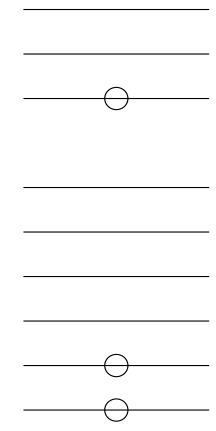

(b)

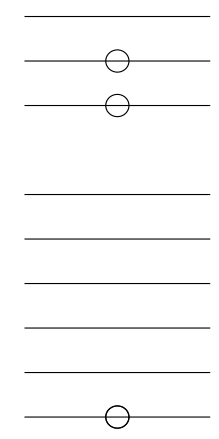

(c)

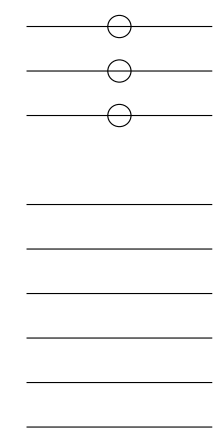

(d)

Figure 1: The figure shows, in the specific case $n=3$ and $\Omega=9$, the partition of the $\Omega$ levels in the set I (with $\Omega-n=6$ levels) and II (with $n=3$ levels).

To this first set correspond $d_{0}=\left(\begin{array}{c}\Omega-n \\ n\end{array}\right)$ configurations. To the second set are associated configurations with $n-1$ pairs in I and one pair in II (see Fig. 1b), their number being $d_{1}=n\left(\begin{array}{c}\Omega-n \\ n-1\end{array}\right)$. In general the $(k+1)$-th set embodies configurations with $n-k$ pairs in I and $k$ pairs in II, their number being

$$
d_{k}=\left(\begin{array}{l}
n \\
k
\end{array}\right)\left(\begin{array}{l}
\Omega-n \\
n-k
\end{array}\right) \quad \text { with } 0 \leq k \leq n \quad(k \text { integer }) .
$$

Clearly $\sum_{k=0}^{n} d_{k}=\left(\begin{array}{l}\Omega \\ n\end{array}\right)$.

With this organization of the levels the matrix (24) splits into $(n+1)^{2}$ rectangular blocks $B_{k j}$ (with $0 \leq k, j \leq n$ ) of dimension $d_{k} \times d_{j}$ and, since the pairing Hamiltonian only connects states differing by the quantum number of one pair, the blocks with $|k-j| \geq 2$ will have vanishing elements. The matrix thus becomes block-tridiagonal.

This suggests the introduction, in lieu of (10), of the following orthonormal set of $n+1$ even commuting variables (the composite "bosons")

$$
\Phi_{k}^{*}=\frac{1}{\sqrt{d_{k}}} \sum_{m=1+s_{k-1}}^{s_{k}}\left[\varphi_{m_{1}}^{*} \ldots \varphi_{m_{n}}^{*}\right]_{m}
$$


where $k$ varies as in (25), $s_{j}=\sum_{l=0}^{j} d_{l}$, being $s_{-1}=0$ and $m$ again identifies the set $\left\{m_{1}, m_{2} \cdots m_{n}\right\}$. Note that the states (26), unlike those in (10), have in general an index of nilpotency higher than one.

The definition (26) also reflects our desire to have the composite bosons retain as much as possible of the symmetry of $H_{P}$. And indeed the $\Phi_{k}^{*}$, while not fully symmetric with respect to the interchange of the $\varphi^{*}$, turn out to be invariant with respect to the interchange of the $\varphi^{*}$ belonging either to set I or to set II. This symmetry property stems from our choice (26) which enforces the maximum coherence among the components of $\Phi_{k}^{*}$. It is remarkable that composite variables corresponding to different combinations of the $\varphi_{m_{1}}^{*} \varphi_{m_{2}}^{*} \cdots \varphi_{m_{n}}^{*}$ not only hold a lower symmetry than the one displayed by (26), but may also lead to the wrong eigenvalues, as we have verified in some instances.

Now in the minimal basis (26) $\mathcal{H}_{P}$ is represented by a $(n+1) \times(n+1)$ matrix whose generic element $\left(M_{n}\right)_{k i} \equiv<\Phi_{k}^{*}\left|\mathcal{H}_{P}\right| \Phi_{i}^{*}>$ results from summing the elements of the block $B_{k i}$ of the $\left(\begin{array}{l}\Omega \\ n\end{array}\right) \times\left(\begin{array}{l}\Omega \\ n\end{array}\right)$ matrix, but for the normalization factor $1 / \sqrt{d_{k} d_{i}}$. The sum is performed by recognizing that all of the blocks have the same number of ones in each row. Specifically, in the diagonal block $B_{k k}$ there are $(n-k)(\Omega-2 n+2 k)$ ones in each row. In the upper diagonal block $B_{k, k+1}$ each row instead contains

$$
c_{k}=(n-k)^{2}
$$

ones. Since the total number of ones in each row of the matrix (24) is $n(\Omega-n)$, the number of ones in the rows of the lower diagonal block $B_{k, k-1}$ will be

$$
b_{k}=k(\Omega-2 n+k) .
$$

As a consequence, the non-vanishing elements of the matrix $M_{n}$ turn out to be

$$
\begin{aligned}
& \left(M_{n}\right)_{k, k+1}=<\Phi_{k}^{*}\left|\mathcal{H}_{P}\right| \Phi_{k+1}^{*}>=\sqrt{\frac{d_{k}}{d_{k+1}}} c_{k} \\
& \left(M_{n}\right)_{k+1, k}=<\Phi_{k+1}^{*}\left|\mathcal{H}_{P}\right| \Phi_{k}^{*}>=\sqrt{\frac{d_{k+1}}{d_{k}}} b_{k+1}
\end{aligned}
$$

and

$$
\left(M_{n}\right)_{k k}=<\Phi_{k}^{*}\left|\mathcal{H}_{P}\right| \Phi_{k}^{*}>=a_{k} \equiv \mathcal{E}+n+n(\Omega-n)-b_{k}-c_{k} .
$$


Clearly $\left(M_{n}\right)_{k, k+1}=\left(M_{n}\right)_{k+1, k}$, since the operator $\mathcal{H}_{P}$ is Hermitian and the basis (26) is orthonormal. The matrix thus becomes tridiagonal, reading

$M_{n}=\left(\begin{array}{ccccccc}a_{0} & \sqrt{\frac{d_{0}}{d_{1}}} c_{0} & 0 & \cdot & \cdot & \cdot & \cdot \\ \sqrt{\frac{d_{0}}{d_{1}}} c_{0} & a_{1} & \sqrt{\frac{d_{1}}{d_{2}}} c_{1} & 0 & \cdot & \cdot & \cdot \\ 0 & \sqrt{\frac{d_{1}}{d_{2}}} c_{1} & a_{2} & \sqrt{\frac{d_{2}}{d_{3}}} c_{2} & 0 & \cdot & \cdot \\ \cdot & \cdot & \cdot & \cdot & \cdot & \cdot & \cdot \\ \cdot & 0 & \sqrt{\frac{d_{k-1}}{d_{k}}} c_{k-1} & a_{k} & \sqrt{\frac{d_{k}}{d_{k+1}}} c_{k} & 0 & \cdot \\ \cdot & \cdot & \cdot & \cdot & \cdot & \cdot & \cdot \\ \cdot & \cdot & \cdot & \cdot & 0 & \sqrt{\frac{d_{n-1}}{d_{n}}} c_{n-1} & a_{n}\end{array}\right)$

and the associated eigenfunctions should be expanded in terms of the composite variables (26), namely

$$
\psi\left(\Phi^{*}\right)=\sum_{k=0}^{n} u_{k} \Phi_{k}^{*}=\sum_{k=0}^{n} \sqrt{d_{k}} w_{k} \Phi_{k}^{*},
$$

where the $\sqrt{d_{k}}$ is introduced for convenience. The coefficients of the expansion (32) are then fixed by the eigenvalue equation

$$
M_{n} \vec{u}=0
$$

which, of the $\left(\begin{array}{l}\Omega \\ n\end{array}\right)$ equations for the $\beta$ 's, reduces to $n+1$ equations for the $w$ 's. The associated eigenvalues $\mathcal{E}$ obey the secular equation

$$
D_{n}=\operatorname{det}\left(M_{n}\right)=0,
$$

where $\mathcal{E}$ enters into the diagonal matrix elements $a_{k}$.

Now from the general theory of symmetric tridiagonal matrices one knows that (34) has $n+1$ distinct and real roots and that these are found by applying the recursive relation [0]

$$
D_{n}=a_{n} D_{n-1}-\frac{d_{n-1}}{d_{n}}\left(c_{n-1}\right)^{2} D_{n-2}
$$

which, after some algebra, yields

$$
D_{n}=y(y-\Omega)[y-2(\Omega-1)][y-3(\Omega-2)] \ldots[y-n(\Omega-n+1)],
$$


where

$$
y=n(\Omega-n)+\mathcal{E}+n .
$$

We thus see that $D_{n}$ has all of the zeros of $D_{n-1}$ plus an extra one for $y=n(\Omega-n+1)$.

Moreover (36) allows us to write down for the general solution of (34) the expression

$$
y=p(\Omega-p+1) \quad \text { with } 0 \leq p \leq n .
$$

Hence the well-known formula for the spectrum of the pairing Hamiltonian

$$
\mathcal{E}=-(n-p)(\Omega-n-p+1)
$$

is recovered, the index $p=v / 2$ being linked to the seniority quantum number $v$.

For a tridiagonal matrix, a recursive relation among the eigenvectors components, similar to (35), can also be established. In the specific case of the matrix $M_{n}$ it reads

$$
d_{k} c_{k} w_{k+1}=-d_{k-1} c_{k-1} w_{k-1}-d_{k} a_{k} w_{k}
$$

where again $0 \leq k \leq n$ and quantities with negative indices are meant to be zero.

Thus for the lowest eigenvalue $y=0(p=0$, zero seniority) we have

$$
w_{0}^{(v=0)}=w_{1}^{(v=0)}=\ldots=w_{n}^{(v=0)},
$$

namely the collective state

$$
\psi_{v=0}=\left(\begin{array}{c}
\Omega \\
n
\end{array}\right)^{-1 / 2} \sum_{k=0}^{n} \sqrt{d_{k}} \Phi_{k}^{*}=\left(\begin{array}{c}
\Omega \\
n
\end{array}\right)^{-1 / 2} \sum_{m=1}^{\left(\begin{array}{c}
\Omega \\
n
\end{array}\right)}\left[\varphi_{m_{1}}^{*} \ldots \varphi_{m_{n}}^{*}\right]_{m} .
$$

Indeed in (42) all of the components of the wave function, i.e. the monomials $\left[\varphi_{m_{1}}^{*} \ldots \varphi_{m_{n}}^{*}\right]_{m}$, are coherently summed up. This state obtains for a specific partition of the levels defining the matrix $M_{n}$. However, any other partition would lead to the same result, the weights of the components all being equal. As a consequence the state (42) has zero degeneracy.

Concerning the second eigenvalue $y=\Omega(p=1$, seniority 2$)$, according to Eq. (40) the components of its eigenstate are 


$$
w_{k}^{(v=2)}=\mathcal{N}_{2}\left(k \Omega-n^{2}\right) .
$$

We have found the following expression for the components of the state associated with a generic seniority $v$

$$
\begin{aligned}
w_{k}^{(v)} & =\mathcal{N}_{v} \sum_{j=0}^{v / 2}(-1)^{j} \frac{\left(\begin{array}{c}
k \\
j
\end{array}\right)\left(\begin{array}{c}
v / 2 \\
j
\end{array}\right)\left(\begin{array}{c}
\Omega-v / 2+1 \\
j
\end{array}\right)}{\left(\begin{array}{c}
n \\
j
\end{array}\right)^{2}}= \\
& =\mathcal{N}_{v}{ }_{3} F_{2}\left(-k,-\Omega+\frac{v}{2}-1,-\frac{v}{2} ;-n,-n ; 1\right)
\end{aligned}
$$

${ }_{3} F_{2}$ being a generalized hypergeometric function. In the above the binomial $\left(\begin{array}{l}k \\ j\end{array}\right)$ is meant to vanish when $j>k$.

In particular, for the vector of the maximum seniority $\vec{w}^{(v=2 n)}$, corresponding to $y=n(\Omega-n+1)(p=n$, seniority $2 n)$, one has

$$
w_{k}^{(v=2 n)}=\mathcal{N}_{2 n}\left[(-1)^{k} \frac{(\Omega-2 n+k) !(n-k) !}{(\Omega-2 n) !}\right] .
$$

In (43), (44) and (45) $\mathcal{N}_{2}, \mathcal{N}_{v}$ and $\mathcal{N}_{2 n}$ are normalization constants.

It is straightforward to show that the general eigenstates (32) reduce to the expressions (20, 21, 22) for $\Omega=4$ and $n=2$.

It is interesting in the present formalism to recover the following important result: for values of $\Omega$ large with respect to $n$, the number of dominant components in $\vec{w}^{(v)}$ decreases with $v$, reflecting the weakening of collectivity with increasing seniority. Indeed in the limit $\Omega>>n$ the eigenvalues are

$$
y \simeq \frac{v}{2} \Omega
$$

and moreover

$$
c_{k}<<\Omega, \quad \frac{d_{k-1}}{d_{k}} c_{k-1} \simeq k \Omega \quad \text { and } \quad a_{k} \simeq\left(\frac{v}{2}-k\right) \Omega .
$$

By inserting the above limits in (40) the $w_{k}^{(v)}$ components corresponding to the eigenvalues (46) are found to be

$$
w_{0}^{(v)}=w_{1}^{(v)}=\ldots=w_{v / 2-1}^{(v)}=0
$$


and

$$
w_{k}^{(v)}=\frac{\left(\begin{array}{c}
k \\
v / 2
\end{array}\right)}{\left(\begin{array}{c}
n \\
v / 2
\end{array}\right)} w_{n}^{(v)}, \quad k=v / 2, \ldots, n .
$$

We thus see that the state with seniority $v$ in the basis of the $\sqrt{d_{k}} \Phi_{k}^{*}$ and in the large $\Omega$ limit has indeed $v / 2$ vanishing components, the remaining $n-v / 2+1$ components being expressed through (49) via the single component $w_{n}^{(v)}$. In other words, when $\Omega$ is large the collectivity of a state with seniority $v$ decreases as $v$ increases, because its components become fewer and, furthermore, the surviving components are more capably expressed through a single one.

As a last point in this letter we briefly address the problem of the angular momentum of the composite bosons entering in our eigenstates. In this, for the sake of illustration we shall confine ourselves to the cases $n=1$ and $n=2$, and the latter only for $\Omega=4$. For this purpose it is natural to express the simple building blocks (10), the monomials, as superpositions of $\Omega$ composite bosons $B_{J}$ of definite even ${ }^{\dagger}$ angular momentum with vanishing third component, according to

$$
(-1)^{j-m} \varphi_{m}=\sqrt{\frac{2}{\Omega}} \sum_{J}\langle j m, j-m \mid J 0\rangle B_{J} .
$$

Moreover we account for the constraints the nilpotency of the $\varphi$ 's variables induces on the composite variables $B_{J}$. These constraints relate the latter among themselves, and hence the $B_{J}$ are no longer independent.

It then immediately follows that $B_{0}^{*}$, a composite s-boson, coincides with the one pair wave function (17). Similarly, with a suitable choice of the parameters $\beta_{m}$, the $v=2$ state (18) can be made to coincide with $B_{J}^{*}$, with $J=2,4, \cdots 2(\Omega-1)$. This state thus represents a broken pair.

For two pairs in $\Omega=4$, the $v=0$ eigenstate $(20)$ is given by the product of two composite s-bosons (two unbroken pairs), namely

$$
\Psi_{0}(n=2)=B_{0}^{* 2} .
$$

Likewise for the $v=2$ state (21) one has

$$
\Psi_{2}(n=2)=\left(-4 a_{1}-2 a_{2}+a_{3}\right) B_{0}^{*} B_{2}^{*}+
$$

\footnotetext{
†Since the $\varphi_{m}$ are even in $m$, only even $J$ are allowed.
} 


$$
+\left(3 a_{1}-2 a_{2}+8 a_{3}\right) \sqrt{\frac{3}{11}} B_{0}^{*} B_{4}^{*}+\left(2 a_{1}-5 a_{2}-2 a_{3}\right) \sqrt{\frac{7}{11}} B_{0}^{*} B_{6}^{*},
$$

namely a wave function of good angular momentum $(J=2,4,6)$, as it is easily verified by appropriately choosing the parameters.

Up to this point we have been able to express the eigenstates of $H_{P}$ only in terms of $s$ and $d$ composite bosons. This, however, is no longer the case for the $v=4$ eigenstate. Indeed, on the one hand it is remarkable that $B_{0}^{*}$ cancels out in the differences among the $\varphi^{*}$ 's which enter into (22), an occurrence to be expected since the state $v=4$ describes two broken pairs. On the other hand, from its explicit expression in term of $B_{J}$, namely

$$
\begin{aligned}
& \Psi_{4}(n=2)=\frac{1}{7}\left(5 b_{3}-4 b_{2}\right) B_{2}^{* 2}-\frac{5}{77}\left(11 b_{3}+b_{2}\right) B_{4}^{* 2}+\frac{7}{11} b_{2} B_{6}^{* 2}+ \\
& -\frac{8}{7 \sqrt{33}}\left(5 b_{3}-4 b_{2}\right) B_{2}^{*} B_{4}^{*}-\frac{4}{\sqrt{77}}\left(b_{3}-3 b_{2}\right) B_{2}^{*} B_{6}^{*}+\frac{4}{11 \sqrt{21}}\left(11 b_{3}+2 b_{2}\right) B_{4}^{*} B_{6}^{*}
\end{aligned}
$$

it appears that (53) cannot be given only in terms of $s$ and $d$ composite bosons. Note that the wave functions discussed here are yet to be normalized.

It is worth pointing out that, since our basis is clearly incomplete insofar as the angular momentum is concerned, in general we are bound to obtain wave functions that contain superpositions of differing angular momenta.

In conclusion the aim of this letter has been to illustrate for the specific example of the pairing hamiltonian, viewed as a prototype for the interaction in complex many-body systems, the suitability of the Grassmann algebra for dealing with composite fields. This approach permits a major reduction in the number of degrees of freedom used to describe many-body systems, in particular to treat the discrete states of nuclei.

The relevant outcomes of the present analysis arise from the building blocks used in setting up our minimal basis. These, in fact,

- keep track of the nature of their constituents through a finite index of nilpotency;

- while not fully symmetric in the exchange of the $\varphi$ 's, nevertheless fulfill a remarkable symmetry property.

Finally, it is seen that more than two composite bosons are required to treat the dynamics of $H_{P}$. 


\section{Acknowledgements}

The authors like to thank Dr. M. Caselle for many useful discussions and Prof. T.W. Donnelly for critical remarks and constructive comments.

\section{References}

[1] F. Iachello and A. Arima, The interacting boson model, (Cambridge University Press, Cambridge, 1897)

[2] M.B. Barbaro, A. Molinari and F. Palumbo, Nucl. Phys. B 487 (1997) 492

[3] S. Caracciolo and F. Palumbo, Nucl. Phys. B 512 (1998) 505

[4] F. Palumbo, Phys. Rev. D 60 (1999) 074009

[5] L.D. Faddeev and A.A. Slavnov, Gauge Fields: Introduction to Quantum Theory (Benjamin, 1980)

[6] P. Ring and P. Shuck, The Nuclear Many Body Problem, (SpringerVerlag, New York, 1980), p. 225

[7] V.V. Prasolov, Problems and Theorems in Linear Algebra, (American Mathematical Society, Providence, 1994), p. 65 